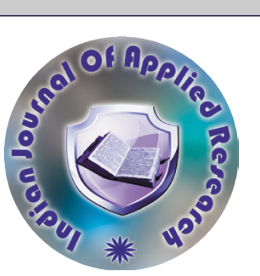

Radiodiagnosis

\title{
EXTENSIVELY DISSEMINATED CYSTICERCOSIS - CASE REPORT AND REVIEW OF LITERATURE
}

\section{Navdeep Kaur* \\ Narinder Kaur \\ Harvinder Singh Chhabra}

Ravinder Kaur
Assistant Professor, Department Of Radiodiagnosis, All India Institute of Medical Sciences, Bathinda, Punjab. *Corresponding Author

Associate Professor, Department Of Radiodiagnosis, Government Medical College \& Hospital, Sector- 32, Chandigarh.

Senior Resident, Department Of Forensic Medicine, GGS Medical College \& Hospital, Faridkot, Punjab.

\section{Professor \& Head, Department Of Radiodiagnosis, Government Medical College \&} Hospital, Sector- 32, Chandigarh.

ABSTRACT

Cysticercosis is a known disease of the developing world and accounts for the majority of epilepsy cases in these nations. The disseminated form represents a rare presentation of the disease with neurocysticercosis being the most common. Patients may present with neurological complaints of seizures only with no clinical manifestations of muscular pseudohypertrophy or palpable subcutaneous nodules usually found in disseminated form falsely localizing disease to the central nervous system. This case report highlights the fact that despite its rarity a high index of suspicion for disseminated form should be kept in mind in endemic areas and warrants routine screening of all patients for multi-organ involvement who present with multiple lesions in brain parenchyma.

\section{KEYWORDS : Disseminated Cysticercosis, Neurocysticercosis, Seizures.}

\section{INTRODUCTION}

Cysticercosis poses a major health burden in developing nations and is caused by the ingestion of eggs of the pork tapeworm Taenia Solium ${ }^{[1]}$ The usual presentation of this disease is neurocysticercosis which is the most common cause of epilepsy in developing nations ${ }^{[2]}$ Disseminated cysticercosis is a very rare entity caused by simultaneous involvement of the brain, subcutaneous, and muscular tissues by cysticercosis ${ }^{[3,4]}$.

Less than 50 cases have been reported worldwide with the majority of cases being from India ${ }^{[3]}$. Here we report a case of extensively disseminated cysticercosis with simultaneous involvement of the brain parenchyma, scalp, muscles of the face, tongue, soft palate, chest wall, paraspinal, psoas, gluteal, pelvic and limbs. This case report highlights the fact that patients with disseminated cysticercosis may present with only neurological complaints and no other clinical manifestations such as palpable nodules or pseudo muscular hypertrophy which are usually seen in cases of disseminated cysticercosis. The diagnosis of this rare presentation may be made only on imaging.

\section{CASE HISTORY}

A 20year old male patient (from a low-income suburban family of North India) student by profession presented to our tertiary care hospital with complaints of recurrent headache and seizures. He was a known case of seizure disorder for four years and was on treatment for the same at a peripheral primary health care center. His seizures were temporarily controlled by the medication but recurred. On examination, the patient was of average built with a normal physical examination. The patient was referred to our department for MRI imaging of the brain for evaluation of the cause of recurrent seizures. MRI revealed numerous T2 hyperintense cysts with eccentric T2 hypointense scolex in them s/o cysticercosis scattered throughout supratentorial as well as in infratentorial brain parenchyma giving starry sky appearance. No evidence of any perilesional edema was seen. Similar multiple T2 hyperintense cysts with eccentric T2 hypointense scolex in them were seen scattered in the scalp, muscles of the face, tongue, soft palate, chest wall muscles, paraspinal, psoas, gluteal, pelvic and limb muscles signifying disseminated form of cysticercosis. On MRI no lesions were found in extra-axial spaces of the brain, in orbit or within the spinal cord. Screening of the chest and abdomen revealed no lesions in the lungs or abdominal organs. The diagnosis was confirmed by characteristic lesion appearance on imaging and positive $\mathrm{IgG}$ antibody in serum. transmission of this disease in humans. Spread of larvae from the intestine by the portal venous system to different parts of the body such as subcutaneous tissue, skeletal muscles, lungs, brain, eyes, liver, and occasionally heart results in the disseminated form of the disease ${ }^{[5]}$.

Disseminated cysticercosis is a rare manifestation of this common disease ${ }^{[6]}$. A review of 450 cases of cysticercosis by Dixon and Lipscomb revealed only one case of disseminated cysticercosis supporting the rarity of this form ${ }^{[7]}$. The extensive spread of cysticercosis in the human body was probably earliest reported by a British army medical officer posted in India in $1912^{\left[{ }^{[8]}\right.}$. Then in 1926 Priest reported the first case of disseminated cysticercosis in a British soldier who had symptoms of swelling in muscles, epileptic seizures, mental dullness, and diffuse subcutaneous nodules ${ }^{[9]}$.

Clinical symptoms in a patient are determined mostly by cyst location, cyst burden, and reaction of the host. Typical manifestations of disseminated form of cysticercosis include pseudo-muscular hypertrophy $(100 \%)$, palpable subcutaneous nodules $(87 \%)$, seizures $(78 \%)$, and abnormal mentation ${ }^{[6]}$

Pseudo-muscular hypertrophy is commonly seen in the disseminated form ${ }^{[5]}$ Bandyopadhyay et al. described a case of cysticercosis with significant pseudohypertrophy of the calf and shoulder muscles ${ }^{[10]}$. Banu et al. reported a case with diffuse involvement of the skeletal muscles, lungs, subcutaneous tissues, and brain by cysticercosis and presenting with only arthritis. On examination, mild symmetrical hypertrophy of calf muscles was seen ${ }^{[5]}$. But in our case, no evidence of any muscular hypertrophy was seen despite extensive skeletal muscle involvement by cysticercosis. Multiple palpable nodules are seen all over the body due to the widespread dissemination of cysticercosis ${ }^{[11}$ which was also not seen in our case. Our patient presented with only neurological complaints of recurrent headaches and seizures. A similar case of disseminated cysticercosis was reported by Soo Yong Park et al presenting with only headache and recurrent generalized tonic-clonic seizures. No palpable nodules or muscular hypertrophy was seen in this patient ${ }^{[12]}$. No further studies are available to highlight this rare clinical presentation of disseminated cysticercosis because of its relative rarity. Clinical features in our patient were limited only to headache and recurrent seizures with no other clinical manifestations such as pseudo-muscular hypertrophy or palpable subcutaneous nodules which were usually seen in cases of disseminated cysticercosis 
better in localizing cysts in extra-axial spaces of the brain such as in cisterns or ventricles and in assessing response to treatment Typical MRI imaging findings of cysticercosis include a well-defined round T2 hyperintense cyst with eccentric T2 hypointense nodule in it which represents scolex. The presence of scolex in a cystic lesion is virtually diagnostic of cysticercosis ${ }^{[14]}$. In our patient, multiple T2 hyperintense cysts with eccentric T2 hypointense scolex in them were seen scattered throughout brain parenchyma, scalp, muscles of the face, tongue, soft palate, chest wall muscles, paraspinal, psoas, gluteal, pelvic and limb muscles. No perilesional edema was seen around any of the lesions.

In a patient presenting with seizures, the main differential on imaging includes tuberculoma which can also present as multiple lesions showing ring enhancement on post-contrast images. However, demonstration of an eccentric scolex in a cystic lesion is pathognomic of cysticercosis ${ }^{[14]}$. In equivocal cases, MR spectroscopy can be used as a problem-solving tool. Demonstration of dominant lipid peak (0.9-1.3 ppm) with the near absence of other metabolites has high sensitivity and specificity for differentiating tuberculomas from other lesions ${ }^{[1,1,1}$ MR spectroscopy was not done in our case due to the characteristic imaging appearance of the lesion i.e. cyst with eccentric scolex in it that posed no diagnostic dilemma.

Pharmacological management is indicated with cysticidal drugs such as praziquantel and albendazole as they reduce the parasite burden and hasten the death of the cysts ${ }^{[17]}$. Treatment with cysticidal agents may result in severe reactions from enlargement of the cysts, massive release of antigens causing local tissue swelling and generalized anaphylactic reaction ${ }^{[18]}$. Giving steroids before starting cysticidal drugs may reduce the incidence of such adverse reactions
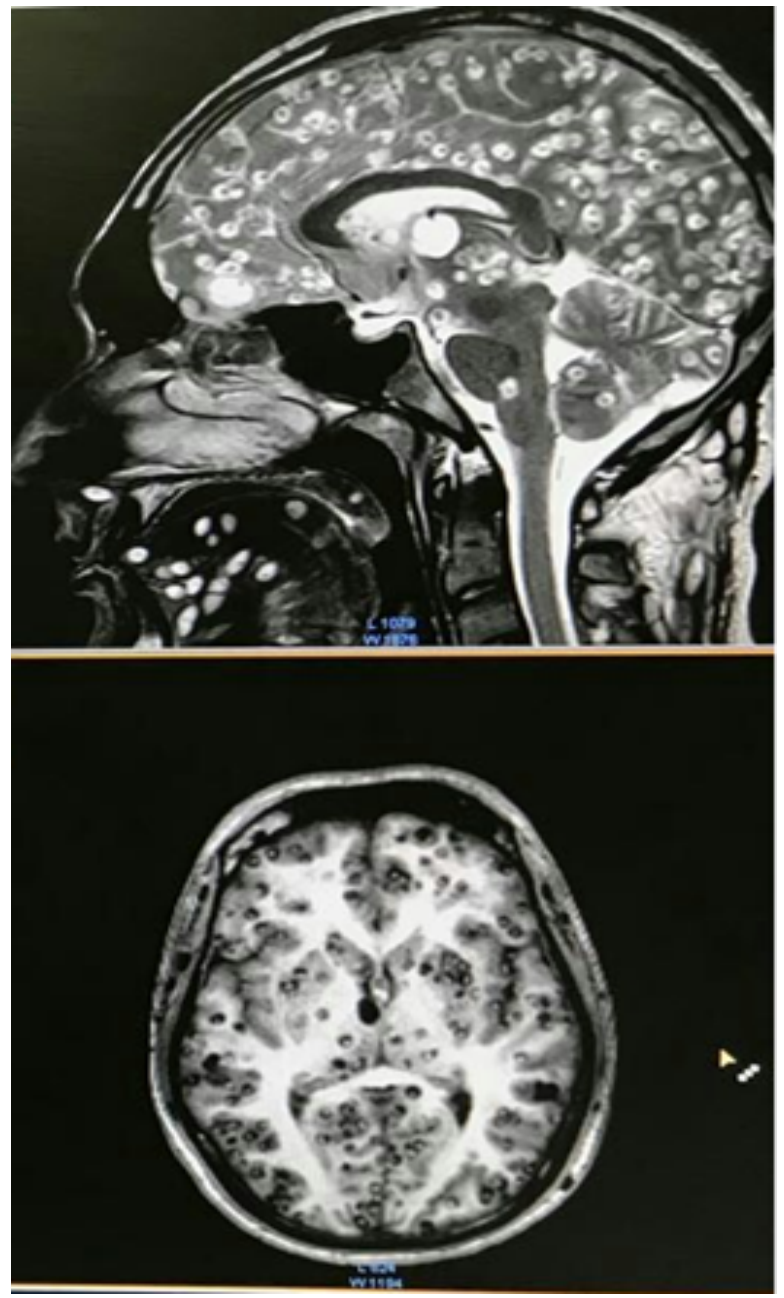

Figure 1. Saggital T2-weighted and Axial T1-weighted MRI images of the brain show multiple cystic lesions with eccentric scolex in them suggestive of cysticercosis diffusely scattered throughout supratentorial as well as infratentorial brain parenchyma, in scalp, muscles of the face, tongue, soft palate and visualized neck muscles.

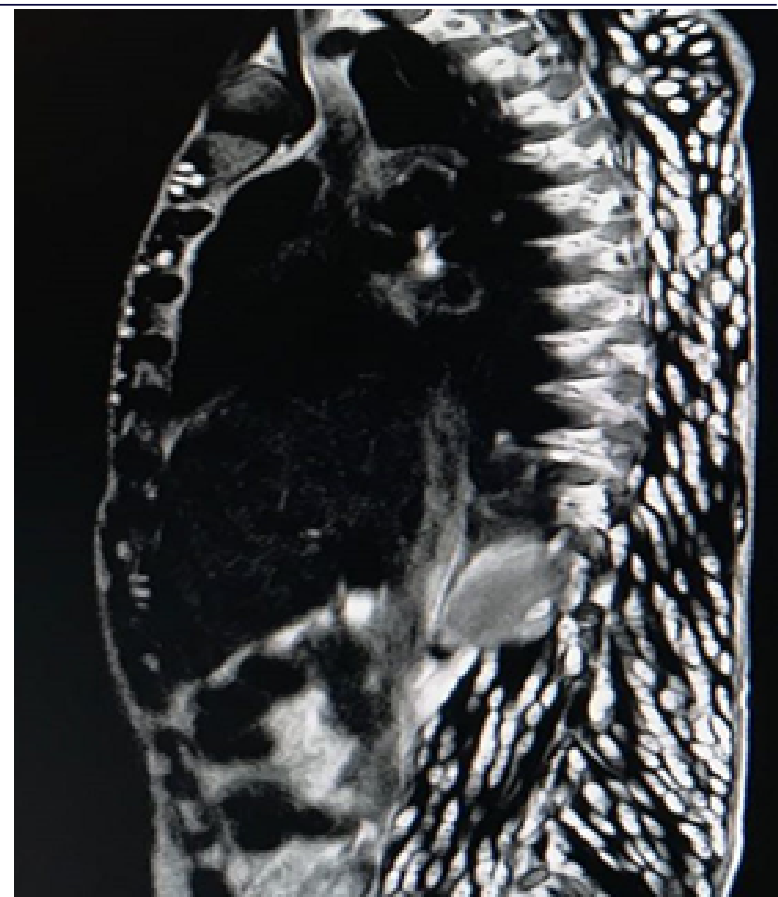

Figure 2. Saggital T2-weighted MRI image of the body shows numerous cysticerci diffusely scattered in paraspinal muscles, psoas muscle, chest wall and abdominal wall muscles.

\section{Acknowledgement: None}

\section{REFERENCES}

Bern C, Garcia HH, Evans C, Gonzalez AE, Verastegui M, Tsang VC, et al. Magnitude of the disease burden from neurocysticercosis in a developing country. Clin Infect Dis 1999;29: 1203-9.

Khandpur S, Kothiwala SK, Basnet B, Nangia R, Venkatesh H A, Sharma R. Extensive disseminated cysticercosis. Indian J Dermatol Venerol Leprol 2014; 80:137-40. Bhalla A, Sood A, Sachdev A, Varma V. Disseminated cysticercosis: a case report and review of the literature. J Med Case Rep 2008; 2:137.

Ming-Pin Lin, Yen-Li Chen, Wen-Sheng Tzeng. Extensive disseminated cysticercosis. BMJ Case Rep 2014

Banu A, Veena N. A rare case of disseminated cysticercosis: Case report and literature Banu A, Veena N. A rare case of disseminated cys

Kumar A, Bhagwani DK, Sharma RK, Kavita, Sharma S, Datar S, et al. Disseminated cysticercosis. Indian Pediatr 1996; 33:337-9.

Dixon HBF, Lipscomb FM. Cysticercosis: An analysis and follow up of 450 cases. Med Res Counc Rep Ser 1961;299:1-58.

8. Krishnaswami CS: Case of Cysticercus cellulose. Ind Med Gaz. 1912; 27:43-44. Wadia N, Desai S, Bhatt M: Disseminated cysticercosis. New observations, including CT scan findings and experience with treatment by praziquantel. Brain.1988;111: 597614.

10. Bandyopadhyay D, Sen S. Disseminated cysticercosis with muscle hypertrophy. Indian JDermatol. 2009; 54:49-51.

11. Dhar M, Ahmad S, Srivastava S, Shirazi N. Disseminated cysticercosis: Uncommon presentation of a common disease. Ann Trop Med Public Health 2013; 6:317-20.

12. Soo Yong Park, Min Ho Kong, Kwan Young Song. J Korean Neurosurg Soc. 2011;49(3): 190-93.

13. Cheung YY, Steinbaum S, Yuh WT, Chiu L: MR findings in extracranial cysticercosis. J Comput Assist Tomogr. 1987; 11:179-181.

14. Del Brutto OH, Rajshekhar V, White AC Jr, Tsang VC, Nash TE, Takayanagui OM, et al. Proposed diagnostic criteria for neurocysticercosis. Neurology. 2001;57: 177-83.

15. Poptani H, Kaartinen J, Gupta RK, Niemitz M, Hiltunen Y, Kauppinen R A. Diagnostic assessment of brain tumours and non-neoplastic brain disorders in vivo using proton nuclear magnetic resonance spectroscopy and artificial neural networks. J Cancer Res Clin Oncol 1999; 125:343-49.

16. Jayasundar R, Singh VP, Raghunathan P, Jain K. Inflammatory granulomas: Evaluation with proton MRS. NMR Biomed 1999;12:139-44.

17. Sotelo J, Escobedo F, Rodriguez CJ, Torres B, Rubio DF: Treatment of parenchymal brain cysticercosis. N Engl J Med. 1984; 310:1001-7.

18. Baily GG: Cysticercosis. Manson's Tropical Disease. Edited by: Cook GC, Zumla A. 2003, London: Saunders, 1584-95. 